\title{
Calculation of Lithium Clearance for Clinical use Based on Renal Excretion in Japanese Patients
}

Takahiro Motoki $^{\star}$, Noriyasu Fukuoka ${ }^{2}$, Kazunori Yamaguchi ${ }^{1}$, Masahiro Watanabe $^{3}$, Hiroaki Tanaka ${ }^{1}$, Takeyoshi Abe ${ }^{4}$, Nariyasu Yoshino ${ }^{2}$, Shinji Kosaka ${ }^{1}$ and Hitoshi Houchi ${ }^{1}$

${ }^{1}$ Department of Pharmacy, Kagawa University Hospital, 1750-1 Ikenobe, Miki-cho, Kita-gun, Kagawa, 761-0793, Japan

${ }^{2}$ Laboratory of Hospital Pharmacy, School of Pharmacy, Nihon University, 7-7-1 Narashinodai, Funabashi, Chiba 274-8555, Japan

${ }^{3}$ Department of Pharmacology, School of Pharmacy, Shujitsu University, 1-6-1 Nishigawara, Naka-ku, Okayama, Okayama 703-8516, Japan

${ }^{4}$ Department of Pharmacy, Social Insurance Ritsurin Hospital, 3-5-9 Ritsurin-cho, Takamatsu, Kagawa 760-0073, Japan

\begin{abstract}
Background: It is recommended to adjust the dosage of lithium carbonate, primarily used in the treatment of mania, based on the serum lithium concentration at steady-state (Css). It has been suggested that factors associated with renal function should be included in the estimation of lithium clearance (Li-CL) due to its elimination via renal excretion. In the present study, parameters affecting Li-CL were investigated in Japanese patients.

Methods: Retrospective analysis was performed in patients who had chronically received lithium carbonate, by stepwise regression analysis, with $\mathrm{Li}-\mathrm{CL}$ as the dependent variable and gender, age, weight and creatinine clearance (Ccr) as independent variables. Ccr was calculated using the Cockcroft-Gault equation, and Li-CL was calculated as the reciprocal value of Css per daily dose.

Results: Seventy-two patients were enrolled in this study. Regression analysis revealed that only Ccr was an independent variable $(\mathrm{P}<0.001)$, and the following equation was obtained: $\mathrm{Li}-\mathrm{CL}(\mathrm{mL} / \mathrm{min})=0.161 \times$ $\mathrm{Ccr}(\mathrm{mL} / \mathrm{min})+6.47$. This equation was then validated by comparison with previously reported methods using a separate population of patients. The bias and precision of the equation's predictions were evaluated by calculating the mean prediction error, mean absolute error and root mean squared prediction error. Although the Jermain method had the least bias, no significant differences were observed between the present and Jermain methods.

Conclusion: Because the equation of the present study includes Ccr as a parameter of renal function, this may better provide appropriate dosing and safety of lithium therapy in Japanese patients.
\end{abstract}

\section{Introduction}

It is well known that both the therapeutic and toxic effects of lithium carbonate, a therapeutic drug for mania and the manic episodes of bipolar disorder, are dependent on lithium blood concentration. The concentration of serum lithium has a narrow therapeutic window of $0.6-1.2 \mathrm{mEq} / \mathrm{L}$, and the risk of toxic symptoms has been reported to appear at concentrations over $1.5 \mathrm{mEq} / \mathrm{L}[1,2]$. It has also been reported that concentration of serum lithium at steady-state (Css) is easily elevated into the toxic range by the patient's conditions and concomitant use of non-steroid anti-inflammatory drugs or diuretics, thereby increasing the risk of lithium intoxication [3]. The Japanese Pharmaceuticals and Medical Devices Agency (PMDA) indicated the possibility that $52 \%$ of patients administered lithium carbonate had never been monitored for Css in Japan [4]. Therefore, periodic measurement of Css was recommended, as well as planning of appropriate administration schedules by therapeutic drug monitoring.

When drug levels are monitored, the information of lithium pharmacokinetic parameters is essential for determination of the optimal dosage. Administration of the "interview form" of lithium carbonate in healthy volunteers showed a renal lithium clearance ( $\mathrm{Li}-$ $\mathrm{CL}$ ) of $0.6 \mathrm{~mL} / \mathrm{kg} / \mathrm{min}$ [5]. Higuchi et al. presented an equation for Li-CL, calculating a clearance of $0.021 \mathrm{~L} / \mathrm{h} / \mathrm{kg}$ based on a report by Mason et al. $[6,7]$. This equation has been frequently used as the initial parameter for the Bayesian estimation. Although these clearance rates are estimated using body weight, factors reflecting the renal function such as age and creatinine clearance (Ccr) are also necessary in the calculation of Li-CL to account for the renal elimination of lithium. Clericetti and Beretta-Piccoli reported that decrease in Li-CL was observed in patients with renal disease whose glomerular filtration

\section{Publication History:}

Received: March 17, 2016

Accepted: May 23, 2016

Published: May 25, 2016

\section{Keywords:}

Lithium clearance, Renal excretion, Pharmacokinetic parameter, Therapeutic drug monitoring rate was decreased, indicating that Li-CL is closely correlated with renal function [8].

Pepin et al. and Jermain et al. reported different methods predicting Li-CL which included Ccr, yet these studies were performed with non-Japanese subjects $[9,10]$. Although Yukawa et al. reported a prediction equation for $\mathrm{Li}-\mathrm{CL}$ based on population pharmacokinetic analysis of Japanese subjects [11], there have been few reports comparing the precision of these prediction equations within the Japanese population.

As discussed above, while Li-CL may be better estimated when including a patient's renal excretion function, package insert of lithium carbonate mentions weight based calculations of clearance instead of renal excretion. Although weight based Li-CL is similar to that based on serum creatinine level (Scr) in patients with normal renal function, the former is overestimated than the latter in patients with abnormal renal function. Consequently, clinicians may unintentionally administer excessive dose possibly leading to adverse reactions. These situations may be the underlying reason why the PMDA imitated the request for lithium

"Corresponding Author: Dr. Takahiro Motoki, Department of Pharmacy, Kagawa University Hospital, 1750-1 Ikenobe, Miki-cho, Kita-gun, Kagawa, Japan; E-mail: tmotoki@med.kagawa-u.ac.jp

Citation: Motoki T, Fukuoka N, Yamaguchi K, Watanabe M, Tanaka H, et al. (2016) Calculation of Lithium Clearance for Clinical use Based on Renal Excretion in Japanese Patients. Int J Clin Pharmacol Pharmacother 1: 107. doi: https://doi. org/10.15344/2456-3501/2016/107

Copyright: (c) 2016 Motoki et al. This is an open-access article distributed under the terms of the Creative Commons Attribution License, which permits unrestricted use, distribution, and reproduction in any medium, provided the original author and source are credited. 
Citation: Motoki T, Fukuoka N, Yamaguchi K, Watanabe M, Tanaka H, et al. (2016) Calculation of Lithium Clearance for Clinical use Based on Renal Excretion in Japanese Patients. Int J Clin Pharmacol Pharmacother 1: 107. doi: https://doi.org/10.15344/2456-3501/2016/107

Page 2 of 5

level monitoring. In this study, we examined the factors affecting the estimation of $\mathrm{Li}-\mathrm{CL}$, and presented an equation predicting Li-CL to utilize in drug design and to avoid over dosage in Japanese patients. Our equation was also evaluated for predictability in comparison with those equations previously reported.

\section{Methods}

\section{Patients}

Patients who had been receiving doses of lithium carbonate $\left(\right.$ LIMAS $\left.^{\circ}\right)$ were inclusively selected at both Kagawa University Hospital and Kagawa Prefectural Marugame Hospital from January 2011 to July 2012. A total of 72 patients consisting of 42 males and 30 females were eligible during the study period. Among these patients, 13 and 59 patients were sampled during the inpatient and outpatient periods, respectively. Patients who were considered ineligible because of medication non-adherence were excluded in this population. None of the patients were administered any drugs which may affect lithium kinetics. Data on age, sex, weight, SCr, daily doses of lithium carbonate and Css were collected. Css was measured no earlier than 5 days after administration, at which time serum lithium concentrations reached a steady state. Ccr was calculated using the Cockcroft-Gault equation [12]. When Scr was $<0.6 \mathrm{mg} / \mathrm{dL}$, the value was corrected to $0.6 \mathrm{mg} /$ $\mathrm{dL}$.

\section{Lithium assay}

The package insert included with the lithium carbonate recommends planning lithium administration based on the lithium trough level [13]. Css was measured at trough level and detected by the flame ionization method.

\section{Data analysis}

Correlation of Li-CL was evaluated by stepwise multiple regression analysis. Multiple regression analysis was performed with the dependent variable as $\mathrm{Li}-\mathrm{CL}$ and the independent variables as gender, age, weight and Ccr. Li-CL was calculated as the reciprocal of concentration per daily dose (C/D): 1/(C/D). SPSS ${ }^{\circledR}$ statistics 17.0 (SPSS Inc.) was used for all statistical analyses. $\mathrm{P}<0.05$ was considered to be significant. This study was approved by the ethics committee of Kagawa University Hospital.

\section{Validation of obtained equation}

The bias and precision in prediction were compared between the present equation and those previously reported. Another population of 44 patients was used to calculate $\mathrm{Li}-\mathrm{CL}$. The following five methods were used in the prediction of $\mathrm{Li}-\mathrm{CL}(\mathrm{mL} / \mathrm{min})$ :

- Higuchi method [6]

$\mathrm{Li}-\mathrm{CL}=0.35 \times \mathrm{TBW}(\mathrm{kg})(\mathrm{TBW}$ : total body weight $)$

- Pepin method [9]

$$
\mathrm{Li}-\mathrm{CL}=0.235 \times \mathrm{Ccr}(\mathrm{mL} / \mathrm{min})
$$

Although ideal body weight (IBW) is originally used for calculation of Ccr in Pepin method, Patrias and Moore reported that substitution of IBW with TBW had no significant effects on the use of this equation for prediction [14]. Therefore, in the present study, TBW was utilized in all calculations of Ccr with the Cockcroft-Gault equation.

- Jermain method [10]

$\mathrm{Li}-\mathrm{CL}=0.155 \times \mathrm{LBW}+0.0885 \times \mathrm{Ccr}(\mathrm{mL} / \mathrm{min})$

$\{$ LBW: lean body weight $=\mathrm{a} \times \mathrm{TBW}(\mathrm{kg})-\mathrm{b} \times[\mathrm{TBW}(\mathrm{kg}) / \mathrm{H}$
(H: height)

Where, male: $\mathrm{a}=1.10, \mathrm{~b}=128$ and female: $\mathrm{a}=1.07, \mathrm{~b}=148$.

- Yukawa method [11]

$$
\mathrm{Li}-\mathrm{CL}=21.9+[0.156 \times \mathrm{TBW}(\mathrm{kg})-5.41] / \mathrm{Scr}(\mathrm{mg} / \mathrm{dL})
$$

- $[0.440 \times($ age -50$)] \times$ a

Where, age (years) $<50: \mathrm{a}=0, \geq 50: \mathrm{a}=1$.

- Abou-Auda method [15]

$\mathrm{Li}-\mathrm{CL}=\mathrm{a}+\mathrm{b} \times \mathrm{Ccr}(\mathrm{mL} / \mathrm{min})$

Where, inpatients: $\mathrm{a}=15.53, \mathrm{~b}=0.185$ and outpatients: $\mathrm{a}=17.02$, $\mathrm{b}=0.141$.

The bias of prediction was calculated as the mean prediction error (ME). The precision of prediction was calculated as the mean absolute prediction error (MAE) and root mean squared prediction error (RMSE). The equations are described below:

$$
\begin{aligned}
& M E=\frac{\sum_{i=1}^{n}\left(X_{i}^{\prime}-X_{i}\right)}{n} \\
& M A E=\frac{\sum_{i=1}^{n}\left|X_{i}^{\prime}-X_{i}\right|}{n} \\
& R M S E=\sqrt{\frac{\sum_{i=1}^{n}\left(X_{i}^{\prime}-X_{i}\right)^{2}}{n}}
\end{aligned}
$$

Where $X^{\prime}$ is the predicted value calculated by the methods discussed above, $X$ is the observed value and $\mathrm{n}$ is the total number of subjects. The $95 \%$ confidence interval (CI) of ME and MAE were calculated. A 95\% CI of ME not containing zero was judged as having a significant bias.

\section{Results}

Table 1 shows the eligible subject characteristics. Daily dose of lithium carbonate and Css were $594.4 \pm 246.0 \mathrm{mg} /$ day and $0.59 \pm$ $0.27 \mathrm{mmol} / \mathrm{L}$, respectively. Stepwise regression revealed that only Ccr was an independent variable (Table 2). Figure 1 shows the correlation between $\mathrm{Li}-\mathrm{CL}$ and $\mathrm{Ccr}$, and the equation obtained is described below (coefficient of determination $\mathrm{R} 2=0.326, \mathrm{P}<0.001$ )

\begin{tabular}{|l|l|}
\hline Parameters & Values (range) \\
\hline Patients & 72 \\
\hline Male/Female & $42 / 30$ \\
\hline Age (year) & $51.7 \pm 17.0(23-80)$ \\
\hline Weight $(\mathrm{kg})$ & $59.3 \pm 13.8(36.0-90.0)$ \\
\hline SCr $(\mathrm{mg} / \mathrm{dL})$ & $0.80 \pm 0.32(0.60-2.70)$ \\
\hline Ccr $(\mathrm{mL} / \mathrm{min})$ & $90.4 \pm 31.5(28.5-170.4)$ \\
\hline Dosage of lithium carbonate $(\mathrm{mg} /$ day) & $594.4 \pm 246.0(200-1200)$ \\
\hline Li concentration $(\mathrm{mmol} / \mathrm{L})$ & $0.59 \pm 0.27(0.21-1.82)$ \\
\hline
\end{tabular}

Table 1: Patient characteristics.

Data are indicated as the number or mean \pm S.D. If $\mathrm{SCr}$ was $<0.6$, the SCr was corrected as the value of 0.6 .

\begin{tabular}{|l|l|l|}
\hline Parameter & $\begin{array}{l}\text { Standardized } \\
\text { partialregression coefficient }\end{array}$ & $P$ value \\
\hline Ccr & 0.571 & $<0.001$ \\
\hline Gender & -0.040 & 0.697 \\
\hline Age & -0.168 & 0.155 \\
\hline Weight & -0.136 & 0.240 \\
\hline
\end{tabular}

Table 2: Stepwise multiple regression analysis for Li-CL.

$P$ values $<0.05$ are considered significant. $\left.(\mathrm{cm})]^{2}\right\}$ 
Citation: Motoki T, Fukuoka N, Yamaguchi K, Watanabe M, Tanaka H, et al. (2016) Calculation of Lithium Clearance for Clinical use Based on Renal Excretion in Japanese Patients. Int J Clin Pharmacol Pharmacother 1: 107. doi: https://doi.org/10.15344/2456-3501/2016/107

Page 3 of 5

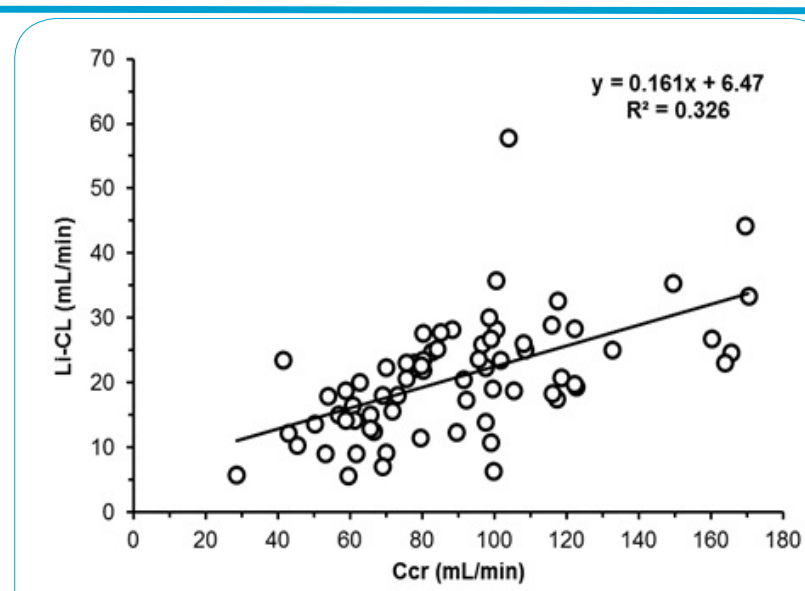

Figure 1: Correlation between Li-CL and Ccr. Data from 72 patients are indicated as opened circle. The solid line represents a linear regression line. Coefficient of determination $\left(\mathrm{R}^{2}\right)$ is $0.326(\mathrm{P}<0.001)$.

$\mathrm{Li}-\mathrm{CL}(\mathrm{mL} / \mathrm{min})=0.161 \times \mathrm{Ccr}(\mathrm{mL} / \mathrm{min})+6.47$

Table 3 shows the Li-CL predicted by previously reported methods, as well as the ME, MAE and RMSE of each method. The range of $95 \%$ CI of ME for the present, Higuchi, Pepin, Yukawa and Abou-Auda methods were greater than zero and only the Jermain method was below zero.

The MAE and RMSE of the present method were similar to those of the previous methods except the Abou-Auda method, with no significant differences among them, although the MAE and RMSE of the Jermain method were the lowest reported values. indicator of renal function in the prediction of Css, as indicated in previous reports $[10,11]$.

Ccr was also the sole parameter affecting Li-CL (Table 2). Li-CL has been reported to be proportional to the glomerular filtration rate or Ccr, at approximately $20-30 \%$ of Ccr $[16,17]$. Pepin et al. reported their Li-CL prediction equation as $23.5 \%$ of Ccr [9], and the present study showed similar results. From these findings, Ccr was speculated to be a suitable parameter of renal function for calculation of Li-CL. In the present study, the correlation between $\mathrm{Li}-\mathrm{CL}$ and Ccr was not high (Figure 1). However, rather than correlation, it may be more informative to evaluate and compare the bias and precision of the different prediction methods, because good correlation does not necessarily indicate an accurate prediction method [18]. Therefore, the evaluation of the present study's prediction method was carried out by comparison with the other previously reported prediction methods.

The present study's prediction equation was evaluated using a separate population of patients. From the analysis of MEs, LiCL tended to be overestimated by all of the equations except the Jermain method, and underestimated only by the Jermain method. In analysis of precision, although the MAE and RMSE of the Jermain method was slightly lower than the other methods, no significant differences were found between the present and the Jermain methods. As a reason for these results, selection of the population of patients used for evaluation is considered to be involved. In the population, obese patients (body mass index $\geq 25$ ) were observed in $61 \%$. In these patients, the Ccr calculated by Cockcroft-Gault equation with TBW was greater, thereby Li-CL predicted by equations using Ccr as a single variable tended to be overestimated. The Jermain method

\begin{tabular}{|l|l|l|l|l|l|}
\hline & Observed Li-CL & Predicted Li-CL & $\begin{array}{l}\text { ME } \\
(95 \% \mathrm{CI})\end{array}$ & $\begin{array}{l}\text { MAE } \\
(95 \% \mathrm{CI})\end{array}$ & RMSE \\
\hline Present study & $18.41 \pm 8.88$ & $22.94 \pm 7.30$ & $\begin{array}{l}4.53 \\
(2.58,6.48)\end{array}$ & $\begin{array}{l}6.21 \\
(4.76,7.66)\end{array}$ & 7.79 \\
\hline Higuchi et al. [6] & $18.41 \pm 8.88$ & $22.66 \pm 5.53$ & $\begin{array}{l}4.25 \\
(2.15,6.36)\end{array}$ & $\begin{array}{l}6.46 \\
(4.97,7.94)\end{array}$ & 8.06 \\
\hline Pepin et al. [9] & $18.41 \pm 8.88$ & $24.04 \pm 10.66$ & $\begin{array}{l}5.63 \\
(3.29,7.98)\end{array}$ & $\begin{array}{l}7.37 \\
(5.54,9.20)\end{array}$ & 9.48 \\
\hline Jermain et al. [10] & $18.41 \pm 8.88$ & $16.13 \pm 4.98$ & $\begin{array}{l}-2.28 \\
(-4.18,-0.38)\end{array}$ & $\begin{array}{l}4.24 \\
(2.70,5.79)\end{array}$ & 6.58 \\
\hline Yukawa et al. [11] & $18.41 \pm 8.88$ & $23.97 \pm 7.86$ & $\begin{array}{l}5.56 \\
(3.17,7.95)\end{array}$ & $\begin{array}{l}7.65 \\
(5.89,9.41)\end{array}$ & 9.56 \\
\hline Abou-Audaet al. [15] & $18.41 \pm 8.88$ & $33.18 \pm 7.63$ & $\begin{array}{l}14.77 \\
(12.49,17.04)\end{array}$ & $\begin{array}{l}15.32 \\
(13.42,17.22)\end{array}$ & 16.52 \\
\hline
\end{tabular}

Table 3: Bias and precision of prediction equations of the present and previous studies.

\section{Discussion}

After absorption, lithium carbonate is transformed into a noncarbonic ion independent of liver metabolism, and is eliminated through the kidneys with sodium and potassium. Therefore, renal function may affect Li-CL, and factors indicating renal function such as age and Ccr are important parameters for its calculation. Therefore, it is necessary to design dosing based on renal function for lithium administration.

From multiple regression analysis, it was shown that Css was dependent on only Ccr, and was correlated with Ccr-based dosage $\left(\mathrm{R}^{2}\right.$ $=0.555$, data not shown). These results suggest that Ccr is a useful includes LBW as a variable and it is the sole prediction method considering correction in somatotype by LBW in this study. Therefore, influence of patient's somatotype might be negligible in the Jermain method. When Li-CL was predicted by Ccr calculated with IBW, the precision of the present and the Pepin methods was improved and equivalent with the Jermain method, suggesting the possibility that correction of body weight by IBW or LBW leads to more precise prediction in extremely obese patients.

The larger MAE and RMSE of the Yukawa method suggest that the present method was more precise than the Yukawa method. Yukawa et al. presented their prediction equation based on analysis 
Citation: Motoki T, Fukuoka N, Yamaguchi K, Watanabe M, Tanaka H, et al. (2016) Calculation of Lithium Clearance for Clinical use Based on Renal Excretion in Japanese Patients. Int J Clin Pharmacol Pharmacother 1: 107. doi: https://doi.org/10.15344/2456-3501/2016/107

Page 4 of 5

of a Japanese population, similar to the present study, and reported that the precision of their method was superior to other methods [11]. Because they reported the precision of their predicted Css and dosage of lithium, there may be a discrepancy in the precision of predicted Li-CL between their and the present studies.

The MAE of the Abou-Auda method was significantly larger than those of the other methods. The Authors reported a high correlation between Li-CL and Ccr, obtained from a stepwise multiple regression analysis in Saudi Arabian subjects [15]. In addition, they reported that the calculation of Li-CL and estimation of lithium dosage using their method was more reliable compared to the Jermain, Pepin and Terao methods [19]. Moreover, Radhakrishnan et al. compared the Pepin, Jermain and Abou-Auda methods for Css prediction, and reported that the Pepin method was the most precise, where as the Abou-Auda method was the least biased [20].

From these evidences, the Abou-Auda method was recommended for use in the prediction of Li-CL. However, in the present study, the Abou-Auda method was the least precise method with the highest MAE and RMSE of all methods. Abou-Auda et al. emphasized the importance of the identification of whether the subject was an inpatient or outpatient for calculation of Li-CL due to differences in life-style factors such as non-compliance, activity and diet. However, this consideration may be negligible in the population of the present study, due to good adherence to therapy schedules as well as generally stable condition of their disease.

Many reports have explored the use of different parameters, such as IBW, TBW, LBW, Ccr and Scr, for substitution of Li-CL. Eq. (1) postulated by Higuchi et al. based on a report by Mason et al. as well as Eq. (2) postulated by Pepin estimates Li-CL as a function of one variable, either TBW or Ccr, respectively. In Eq. (2), Ccr is calculated using IBW, not TBW. Eqs. (3) and (4), postulated by Jermain et al. and Yukawa et al., include two variables, Ccr and LBW, and TBW and Scr, respectively. Patients were divided into two groups by gender for LBW, or by age for the other two equations. Eq. (5) postulated by AbouAuda et al. utilizes Ccr, and divides subjects based on their status as an in- or outpatient. While there have been previous evaluations of the precision of these prediction methods [6, 14, 20-22], standardized prediction methods using $\mathrm{Li}-\mathrm{CL}$ and Css remain to be elucidated.

Because kidney weight may be proportional to body weight, use of IBW or LBW may provide a more precise estimation of individual kidney weight than TBW. Normal renal excretion may then be expressed as a function of these parameters. However, eqs. (1) and (4) may not sufficiently correspond to variation of age due to poor information about age in these parameters. Renal excretion of lithium has been reported to gradually decrease as patients become older [23]. These findings suggest that prediction of Li-CL using only body weight may not be appropriate, and that an individual's function may not be estimated by these parameters. Correlation between Scr and renal function has been demonstrated in previous reports, offering more individualized information of $\mathrm{Li}-\mathrm{CL}$, yet estimation of Ccr is sometimes confounded in senior females due to their poor body muscle. The Cockcroft-Gault equation estimates Ccr by three parameters: age, body weight and Scr. Furthermore, Ccr may correlate closer with Li-CL fluctuation than the weight-related parameters and Scr. Therefore, from the above findings, eqs. (2)-(5) may be affected by these factors when estimating $\mathrm{Li}-\mathrm{CL}$.

For Japanese subjects, the Yukawa method estimates Li-CL using
TBW and Scr, but divides subjects into two groups according to age. Limitations of the present study include the retrospective analysis of a limited number of patients. However, the present results show that estimation of Li-CL using Ccr without classifying subjects by age or gender is not inferior to the Yukawa method for Japanese patients. Although our results would be reasonably assumed by Li disposition, the present equation may help avoid excessive administration and be clinically useful especially in Japanese patients with abnormal renal function.

\section{Conclusion}

In conclusion, although body weight remains to be used for the determination of lithium dosage in accompanying package inserts, our results suggested that $\mathrm{Ccr}$ is a more suitable parameter for estimation of Li-CL than body weight. Dosage design of lithium administration based on renal function may be useful for the future development of safe and effective therapy.

\section{Competing Interests}

The authors declare that they have no competing interests.

\section{References}

1. Amdisen A (1975) Monitoring of lithium treatment through determination of lithium concentration. Dan Med Bull 22: 277-291.

2. Amdisen A (1977) Serum level monitoring and clinical pharmacokinetics of lithium. Clin Pharmacokinet 2: 73-92.

3. Timmer RT, Sands JM (1999) Lithium intoxication. J Am Soc Nephrol 10: 666-674.

4. Pharmaceutical and Medical Devices Agency (2012) PMDA request for proper use of drugs.

5. Interview Form of LIMAS $₫$ tablets $100 \mathrm{mg}$ and $200 \mathrm{mg}$. Taisho Toyama Pharmaceutical Co., Ltd.

6. Higuchi S, Fukuoka R, Aoyama T, Horioka M (1988) Predicting serum lithium concentration using Bayesian method: a comparison with other methods. J Pharmacobiodyn 11: 158-174.

7. Mason RW, McQueen EG, Keary PJ, James NM (1978) Pharmacokinetics of lithium: elimination half-time, renal clearance and apparent volume of distribution in schizophrenia. Clin Pharmacokinet 3: 241-246.

8. Clericetti N, Beretta-Piccoli C (1991) Lithium clearance in patients with chronic renal diseases. Clin Nephrol 36: 281-289.

9. Pepin SM, Baker DE, Nance KS (1980) Lithium dosage calculation from age, sex, height, weight and serum creatinine. In Proceedings of the 15th Annual ASHP Midyear Clinical Meeting.

10. Jermain DM, Crismon ML, Martin ES 3rd (1991) Population pharmacokinetics of lithium. Clin Pharm 10: 376-381.

11. Yukawa E, Nomiyama N, Higuchi S, Aoyama T (1993) Lithium population pharmacokinetics from routine clinical data: role of patient characteristics for estimating dosing regimens. Ther Drug Monit 15: 75-82.

12. Cockcroft DW, Gault MH (1976) Prediction of creatinine clearance from serum creatinine. Nephron 16: 31-41.

13. Package Insert of LIMAS $®$ tablets $100 \mathrm{mg}$ and $200 \mathrm{mg}$. Taisho Toyama Pharmaceutical Co., Ltd.

14. Patrias JM, Moore FH (1985) Evaluation of two methods for predicting initial lithium dosage without measuring serum concentrations. Clin Pharm 4: 77-79.

15. Abou-Auda HS, Al-Yamani MJ, Abou-Shaaban RR, Khoshhal SI (2008) A new accurate method for predicting lithium clearance and daily dosage requirements in adult psychiatric patients. Bipolar Disord 10: 369-376. 
Citation: Motoki T, Fukuoka N, Yamaguchi K, Watanabe M, Tanaka H, et al. (2016) Calculation of Lithium Clearance for Clinical use Based on Renal Excretion in Japanese Patients. Int J Clin Pharmacol Pharmacother 1: 107. doi: https://doi.org/10.15344/2456-3501/2016/107

16. Higuchi S (1993) Fact of TDM for dug dosage design. Yakugyozihousha Co Ltd, Tokyo 274-292.

17. Thomsen K, Shirley DG (1997) The validity of lithium clearance as an index of sodium and water delivery from the proximal tubules. Nephron 77: 125138.

18. Sheiner LB, Beal SL (1981) Some suggestions for measuring predictive performance. J Pharmacokinet Biopharm 9: 503-512.

19. Terao T, Okuno K, Okuno T, Nakano H, Shinkai T, et al. (1999) A simpler and more accurate equation to predict daily lithium dose. $\mathrm{J}$ Clin Psychopharmacol 19: 336-340.

20. Radhakrishnan R Kanigere M, Menon J, Calvin S, Srinivasan K (2012) Comparison of three a-priori models in the prediction of serum lithium concentration. Indian J Pharmacol 44: 234-237.

21. Stip E, Dufresne J, Boulerice B, Elie R (2001) Accuracy of the Pepin method to determine appropriate lithium dosages in healthy volunteers. $J$ Psychiatry Neurosci 26: 330-335.

22. Wright $\mathrm{R}$, Crismon $\mathrm{ML}$ (2000) Comparison of three a priori methods and one empirical method in predicting lithium dosage requirements. Am J Health Syst Pharm 57: 1698-1702.

23. Hewick DS, Newbury P, Hopwood S, Naylor G, Moody J (1977) Age as a factor affecting lithium therapy. Br J Clin Pharmacol 4: 201-205. 